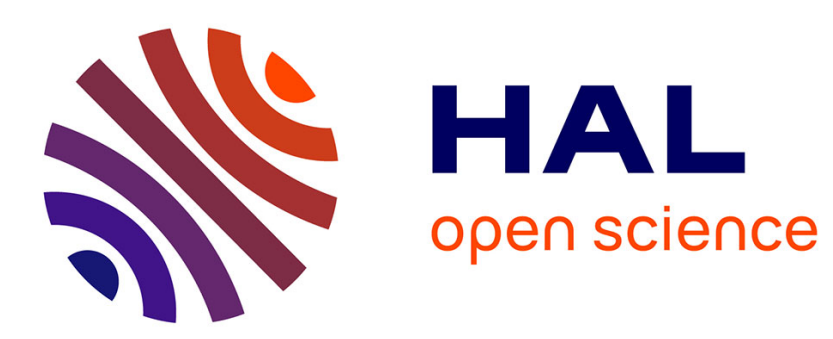

\title{
Computer Supported Interaction Analysis of Group Problem Solving
}

Martin Muehlenbrock, Ulrich Hoppe

\section{To cite this version:}

Martin Muehlenbrock, Ulrich Hoppe. Computer Supported Interaction Analysis of Group Problem Solving. CSCL 1999, 1999, United States. 10 p. hal-00197412

HAL Id: hal-00197412

https://telearn.archives-ouvertes.fr/hal-00197412

Submitted on 14 Dec 2007

HAL is a multi-disciplinary open access archive for the deposit and dissemination of scientific research documents, whether they are published or not. The documents may come from teaching and research institutions in France or abroad, or from public or private research centers.
L'archive ouverte pluridisciplinaire HAL, est destinée au dépôt et à la diffusion de documents scientifiques de niveau recherche, publiés ou non, émanant des établissements d'enseignement et de recherche français ou étrangers, des laboratoires publics ou privés. 


\title{
Computer Supported Interaction Analysis of Group Problem Solving
}

\author{
Martin Mühlenbrock, Ulrich Hoppe
}

University of Duisburg, Department of Mathematics/Computer Science

Abstract: Collaboration is widely regarded as beneficial for learning. Collaboration is the mutual engagement of participants in a coordinated effort to solve a problem together. It has been conclusively argued that a focus on the process of collaboration is necessary in order to understand the value of working together with peers for learning.

In accordance with the approach of open distributed learning environments (ODLEs), we have developed a framework system for computer-supported cooperative learning and working. This distributed shared workspace system is well suited for monitoring and assessing group interaction in co-located and distant scenarios. It provides adaptable mechanisms for the automated micro-analysis of processes of collaboration as well as for visualization and feedback.

The system has been used in determining conflicts in focus setting as well as initiative shifts in aggregation and revision phases during a sample of collaborative sessions on puzzle problem solving.

Keywords: problem-based learning, computer-mediated communication, discourse analysis

\section{Introduction}

Recently, the nature of collaboration for learning and the dynamics of group interactions in learning environments have gained considerable interest (Dillenbourg, Baker, Blaye \& O'Malley, 1995, Baker \& Lund, 1996, Burton, Brna, \& Pilkington, 1997). For single user learning environments, Akhras \& Self (1996) have laid out a formal framework for modeling the process of learning on the basis of a micro-analysis of user-environment interactions. We propose an approach for the detailed assessment of group interactions in a multi-user learning environment.

Since the advent of computer supported collaborative work, the investigation of computer support for collaborative learning has been of major interest. It has been conclusively argued that a focus on the processes of collaboration is necessary in order to understand the value working together with peers for learning. Collaboration is the mutual engagement of participants in a coordinated effort to solve a problem together (Roschelle \& Teasley, 1991).

A number of systems have been developed to support collaborative learning, e.g. by simulation, remediation or information sharing. In principle, these learning environments also offer the opportunity to gain deeper insights in the processes of collaboration by automatically monitoring and analyzing the individual traces of the interaction moves. Presenting abstracted patterns from the collaboration protocol to the collaborators themselves is supposed to stimulate them to reflect on their interaction as well as to provide feedback to tutors. 
environments (ODLEs) first proposed in Mühlenbrock, Tewissen, \& Hoppe (1997), we have developed a framework system for computer-supported cooperative learning and working. This shared workspace system seems well suited for monitoring and assessing group interaction in co-located and distant scenarios. It provides adaptable mechanisms for automated analysis of processes of collaboration as well as for visualization and feedback.

This paper is organized as follows. In the following section, different approaches on the assessment of human-system and computer supported human-human interaction will be discussed. Then we present in more detail the shared workspace system that constitutes the basis for the assessment of collaborative problem solving processes. This will finally be demonstrated by means of a worked out example application.

\section{Assessing collaboration}

It has been argued that developing integrated theories considering also environmental variables such as contextual, physical, and temporal factors could help in understanding and modeling human learning (Vosniadou, 1996). Along these lines, Akhras \& Self $(1996,1999)$ have laid out a formal basis for modeling the process of learning, which is therefore characterized by its context, the activity involved, and its extension over time. Learning is regarded as the construction of knowledge through interacting in a world in which learners experience the domain and interpret their own experiences. For single-user learning environments, functions are formally defined in predicate logic

- for extracting information from the process of the learner interacting with the learning environment,

- for analyzing the process of learner-environment interactions,

- for determining the state of the learning process, and

- for changing the environment to enable changes in the state of the learning process.

The analysis is based upon notions of constructive, cumulative, reflective, and self-regulative courses of interaction. In addition, desired situations are predicted by the affordance of situations with respect to these notions.

Moreover, a repertoire of methods for analyzing user-system interactions has been developed in the field of plan recognition. Lewis (1988) investigated analysis-based generalizations of procedures in the context of human-computer interactions. He defined and empirically evaluated cognitively motivated heuristics such as the loose-ends heuristic. Hoppe (1993) investigated task models on file management that reflect the mapping of some set of meaningful external tasks onto the action primitives of a given device. Supportive functions recognize partial or complete plans for assessing the user's current task context and infer potential user goals. In contrast to the GOMS approach of task analysis (Kieras, 1994), plans can be directly nested.

Though frameworks and techniques for assessing single user interactions with systems already exist, they still have to be developed for multi user task analysis. Recently, work has begun on models of collaborative task-oriented discourse. These models put emphasis on group-oriented notions such as initiative changes (Guinn, 1998) and focus shifts in dialogues (Burton, Brna, \& Pilkington, 1997). Baker \& Lund (1996) investigated the implications of different communication interface layouts on collaboration and problem solving. Moreover,

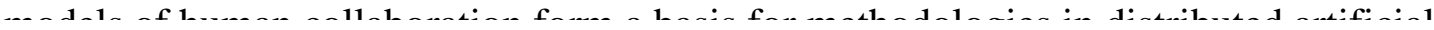


and collaboration mechanisms (see e.g. Huhns \& Singh, 1997).

\section{System architecture}

The framework system is characterized by the provision of opportunities for group interaction, by the combination of intelligent support with interactive learning environments, by the provision of reusable components, and by a distributed multi-agent architecture (Mühlenbrock, Tewissen, \& Hoppe, 1998). The system has been tested with several applications, e.g. for arithmetic exercises, puzzles, microworld robot control, Petri nets, and group discussions. Essentially, the system is composed of two parts, i.e.

- CardBoard user interfaces with distributed shared workspaces and semi-formal visual representations, so-called card nets, and

- CardDalis add-on components for the provision of operational semantics and intelligent support.

In the CardBoard user interface, the user can create several workspaces and freely add, arrange, and modify workspace elements. For each workspace, a specific set of possible elements (cards) is pre-defined (visual language). Cards can also be moved or copied between workspaces, and they can be connected by links to build up card nets. The contents of workspaces can be synchronized between two or more CardBoards for workspace sharing.

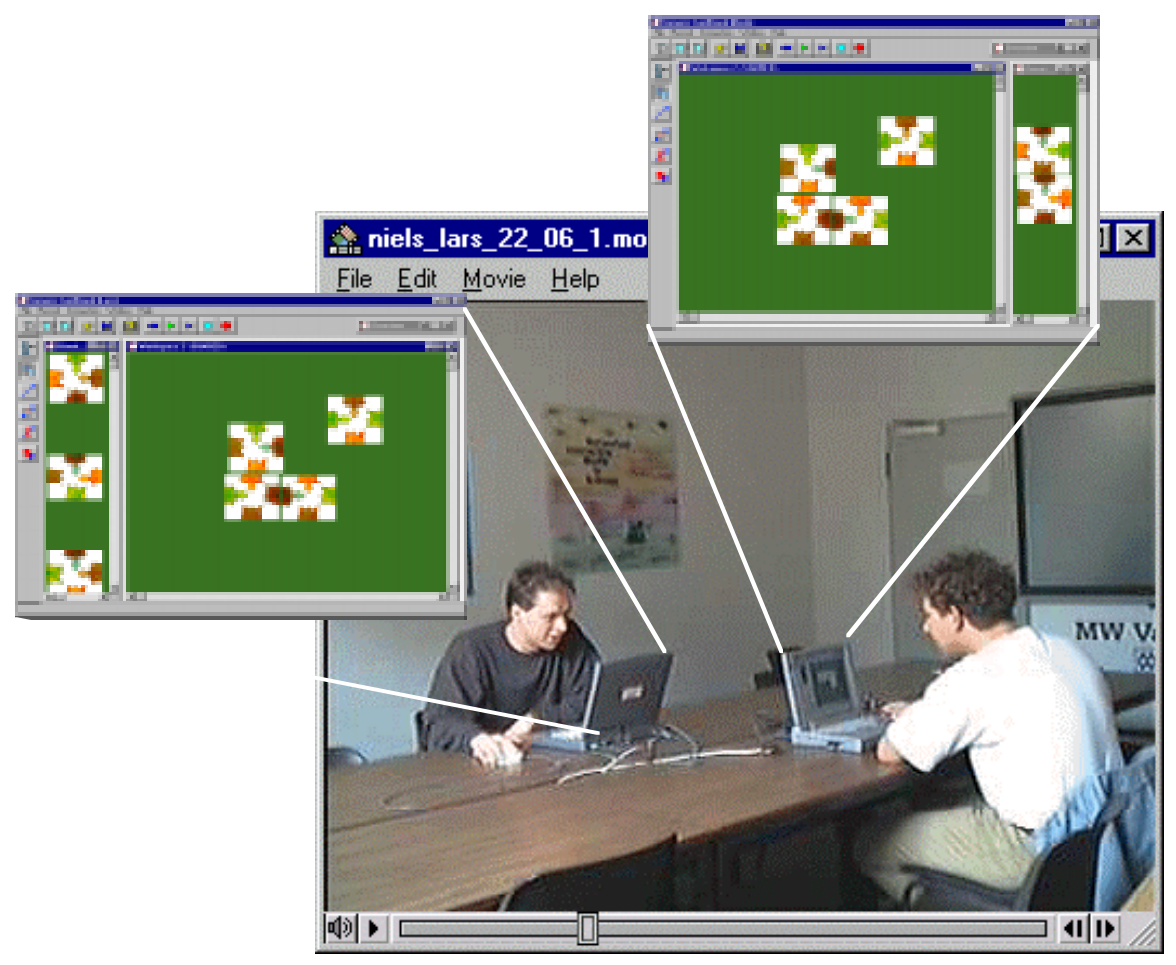

Figure 1: CardBoard user interfaces with shared workspaces.

In the example application in Figure 1, each CardBoard user interface contains three workspaces. The workspace in the center is shared among the two user interfaces. In this example, the task is to solve a turtle puzzle, i.e., to drag cards from the private palette workspaces to the shared workspace and to arrange the cards in a way to have matching turtles in color and shane. The problem can onlv be solved coonerativelv. since the limited set 
1 shows an intermediate step of the problem solving process, where each player has dragged some cards from the private workspace on the left of his or her CardBoard to the shared workspace in the middle and both users have arranged the four cards in order to solve the puzzle.

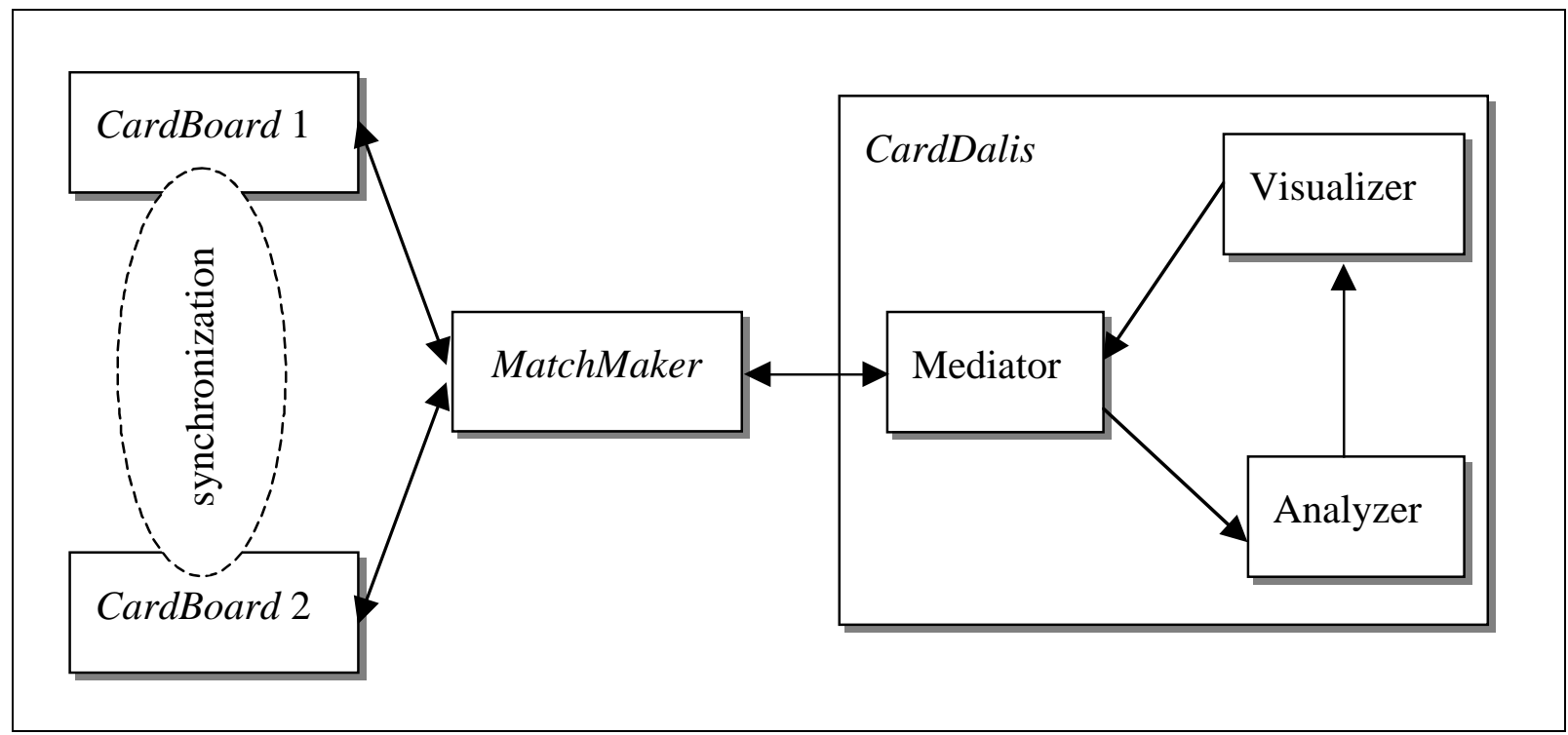

Figure 2: Architecture of the framework system.

The synchronization of shared workspaces is achieved by a replicated architecture facilitated by a synchronization manager called MatchMaker. Figure 2 sketches the architecture of the framework system. In order to synchronize shared workspaces, two or more CardBoards communicate via the central MatchMaker synchronization manager by TCP/IP. The communication is based on a standardized message format for card nets to minimize bandwidth. Each message contains fields for the action that has been performed by an actor on a specific object with an $i d$ and possibly further arguments (see Table 1).

\begin{tabular}{|l|l|l|l|l|}
\hline action & general & object & obligatory & additional \\
\hline \hline $\begin{array}{l}\text { create } \\
\text { modify } \\
\text { delete }\end{array}$ & $\begin{array}{l}\text { id } \\
\text { actor }\end{array}$ & application & user, language & \\
\cline { 3 - 5 } & workspace & language & $\begin{array}{l}\text { title, mode, dimension, } \\
\text { visible_area }\end{array}$ \\
\cline { 3 - 5 } & card & $\begin{array}{l}\text { type, position, } \\
\text { workspace }\end{array}$ & $\begin{array}{l}\text { content, position, mark, } \\
\text { exclusivemark, dimension, } \\
\text { shape, color }\end{array}$ \\
\cline { 3 - 5 } & link & $\begin{array}{l}\text { type, connector, } \\
\text { destination }\end{array}$ & \\
\hline
\end{tabular}

Table 1: Message primitives for synchronization of workspace activities.

Further components (agents) for the provision of operational semantics and intelligent support can be added to the workspace environment by a general architecture called Dalis (Mühlenbrock, Tewissen, \& Hoppe, 1998). CardDalis has been specifically developed for the automated interpretation and generation of card workspaces and their contents. Mainly, CardDalis comprises three components, i.e., a mediator, an analyzer, and a visualizer (see Figure 2). The mediator interfaces CardDalis to the CardBoard environment and internally represents and reconstructs the state of the workspace contents on the basis of incoming card 
analyzer takes the reconstructed data as input and derives analysis concepts on the collaboration from this data. The automatic derivation of these concepts is based on explicitly formulated rules and will be described in the next section. The visualizer processes the derived facts from the analyzer to generate further workspaces with cards that present the analysis to the collaborators or a tutor.

\section{Task analysis of collaboration}

In assessing multi user interaction and problem solving, certain aspects are of specific interest. For instance, it is likely that two collaborators have quite different methods of solving a problem at hand. The general idea is to assign collaborators to different roles depending on their initiatives in setting or shifting the focus of the problem solving process and to determine the whether next actions are chosen in accordance with the context, e.g. whether prior actions of collaborators are taken into account or are neglected.

The shared workspace system described in the previous section constitutes on ODLE that is well suited for the analysis of collaborative problem solving. The collaboration process is analyzed on the basis of an online protocol of user actions related to events in card workspaces. For instance in case of the spatial interpretation of cards and their relative positions as in the example application with the turtle puzzle (Figure 1), the analysis derives from the basic message primitives as described in Table 1 and starts with inferring facts on the pairwise adjacency of cards. The adjacency of two cards is inferred from their actual positions and dimensions including some fuzziness, i.e. that adjacent cards may overlap or be apart to a certain degree. Cards that are adjacent to each other are asserted to be in the same cluster. These concepts related to the spatial arrangement of cards form the basis for the analysis of higher level concepts in a derivation hierarchy (see Figure 3). 


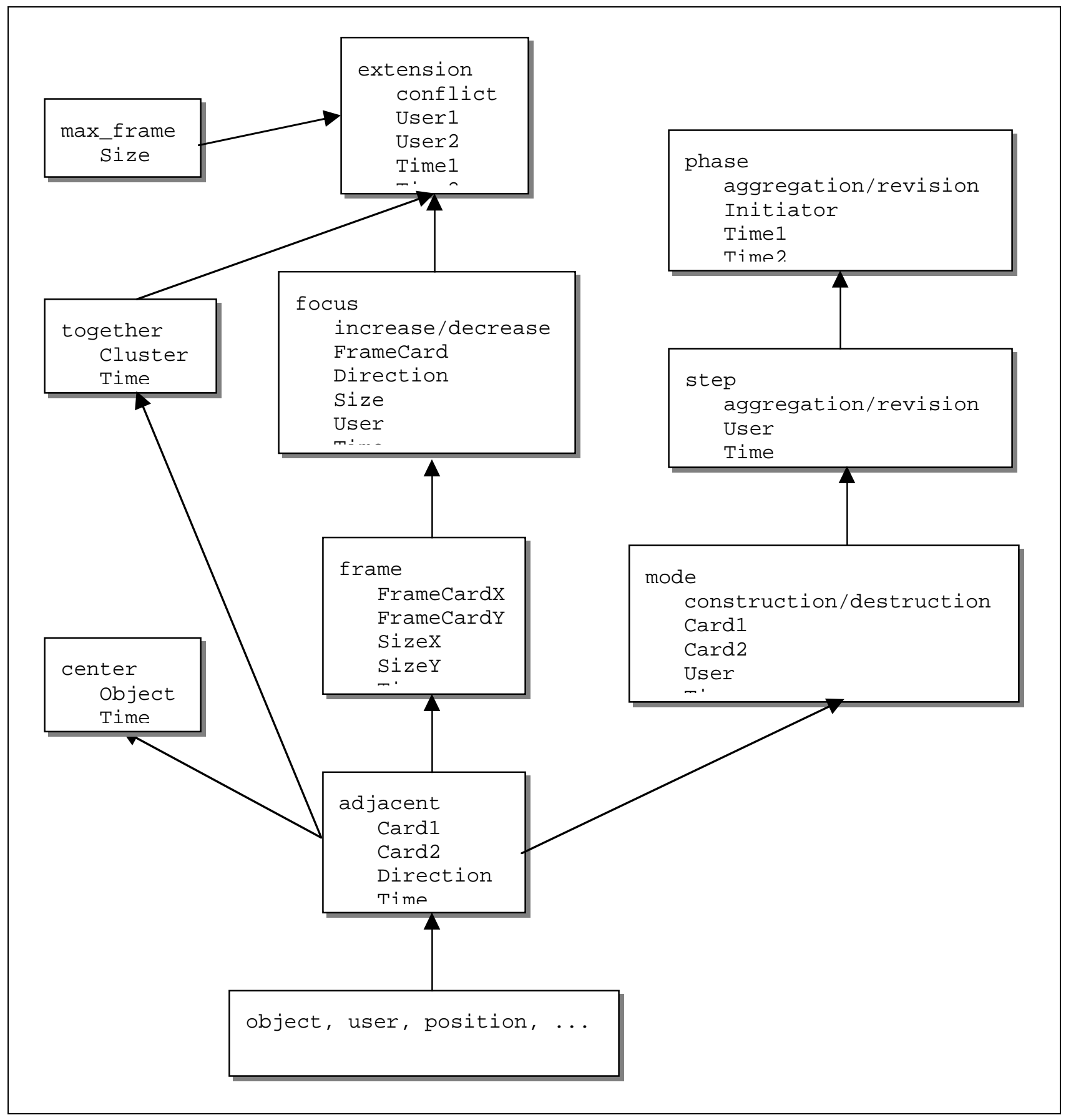

Figure 3: Derivation hierarchy of analysis concepts.

Different problem solving activities such as aggregation, conflict creation, revision, or focus shifting are assigned to the action protocol. For instance, the following rule derives the concept of a conflict in regards of different problem solving foci, e.g. in the turtle puzzle example (cf. also top of Figure 3):

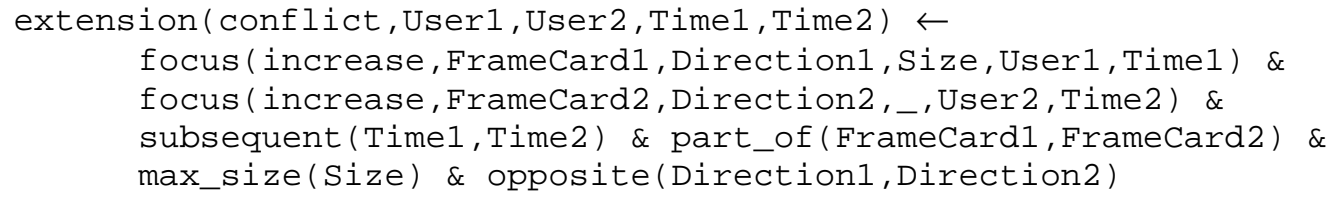

According to this rule, a conflict between two users arises when they subsequently increase the outer extension of the solution. i.e. the arrangement of adiacent turtle cards. bevond a 
is three, since the final puzzle forms a square of three times three cards.

During the online processing of the action protocol, the derived facts are visualized and presented to the users through the creation of additional workspaces by the mediator. Figure 4 shows an intermediate state of a real problem solving session where a focus conflict between the two collaborators has been detected by the rules given above. The analysis workspace presents derived concepts relevant to extensions of the problem solutions that indicate different user foci. The conflict recognized is due to addition of the topmost card by the second user, which contradicts the preceding addition of the bottommost card by the first user in terms of the maximal extension of the solution.

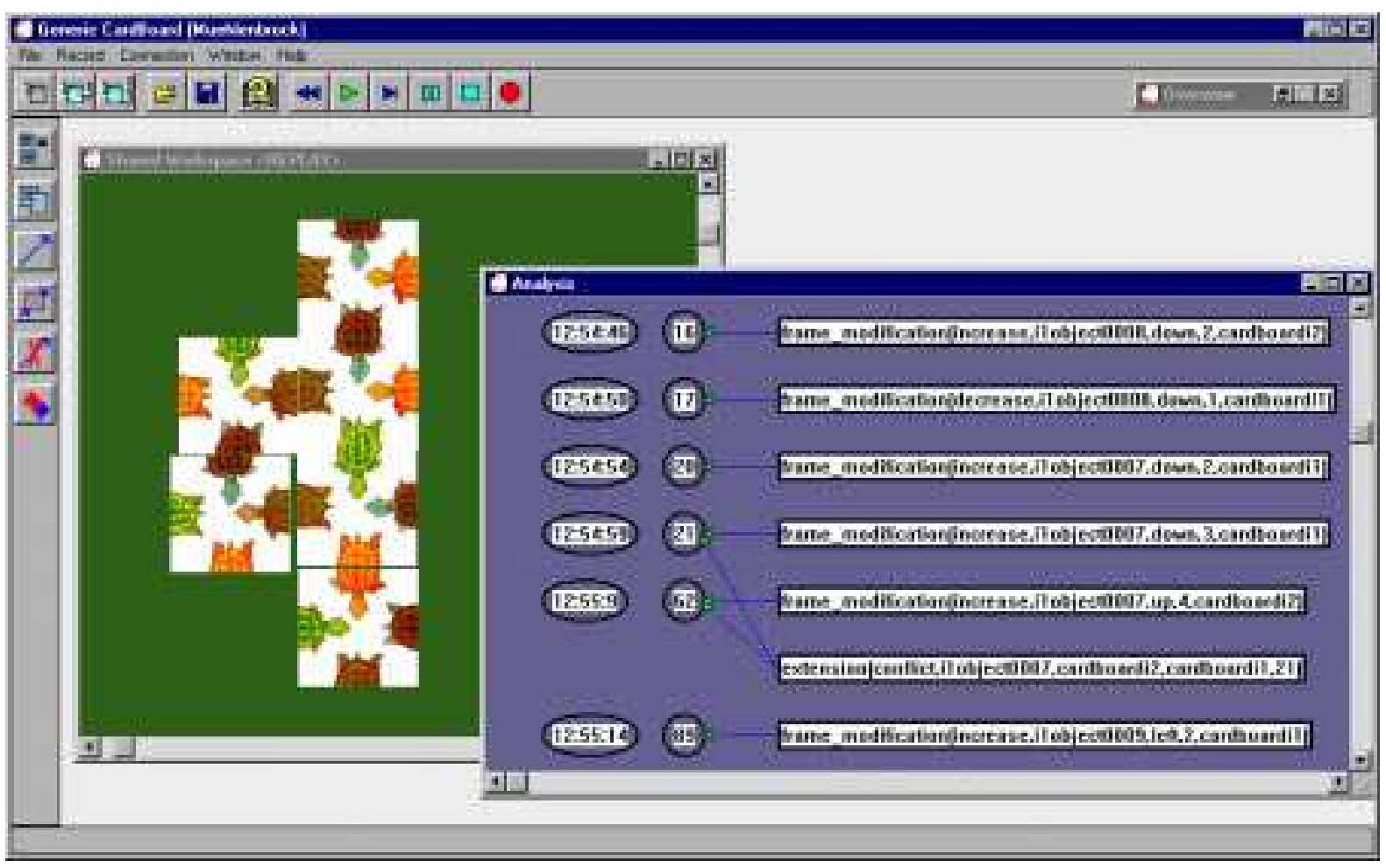

\section{Figure 4: Visualization of analysis concepts in an additional workspace.}

Furthermore, the role each user plays in the process of collaboration can generally be determined by relating initiatives and leading activities of users to problem solving steps. For instance, a user action is classified as a revision step, if the problem solution is modified in a destructive as well as constructive manner. This is usually the case when a user moves a card, which is already part of the solution, to another position to resolve a dead-end. Figure 5 and Figure 6 show the analysis of aggregation and revision steps of different collaborators. The figures have been produced with a standard spreadsheet tool directly from the data that has been generated in table format by the visualizer on the analysis. 


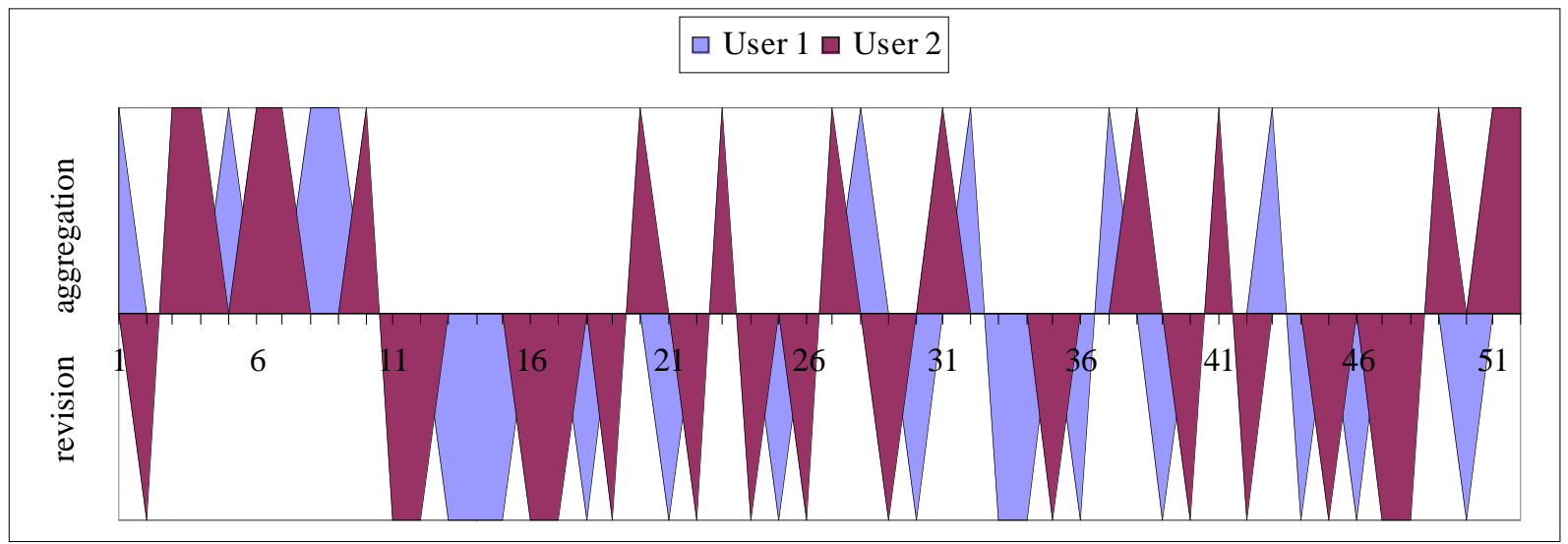

Figure 5: Aggregation and revision steps in first session of collaborative problem solving.

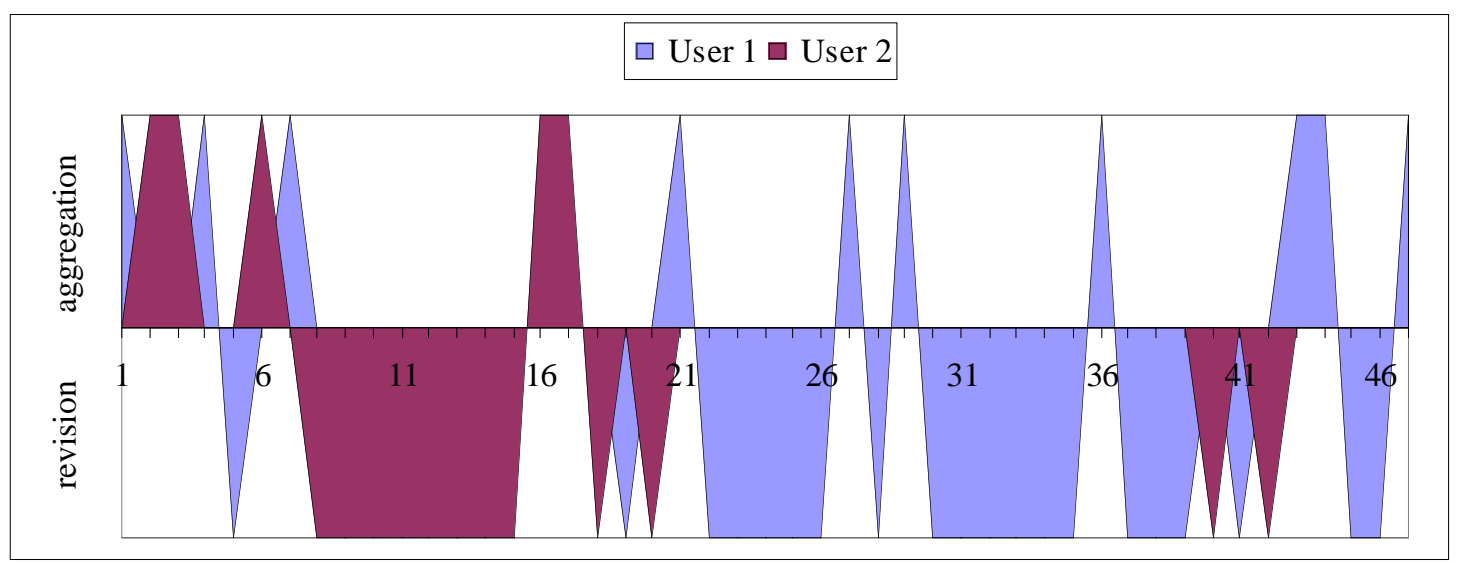

Figure 6: Aggregation and revision steps in second session of collaborative problem solving.

Both example collaborations start with a phase of aggregation steps initiated by user 1, followed by a phase of revision steps initiated by user 2 . After these similar initial phases, phases of altering aggregation and revision steps occur, which are different in the two examples. Whereas in the first example, both users frequently take the initiative, in the second example the initiative remains with user 1 for the most part and aggregation and revision phases alter less frequently.

\section{Conclusions and further work}

In this paper, we have presented an approach and a system for the task-oriented analysis of collaborative problem solving. The framework system has been implemented in $\mathrm{C}^{++}$and Prolog on a heterogeneous system platform with TCP/IP interprocess communication. A tentative set of analysis concepts including their visualization in an overview workspace has been modeled for the turtle puzzle application and has been tested with some collaborative sessions by human users.

In contrast to other approaches to collaboration analysis, the approach presented here is based on task analysis and group interaction is not affected by the need to use interaction markers such as sentence openers (Baker \& Lund, 1996, Soller, Linton, Goodman, \& Lesgold, 1999). Sentence openers rely on text-based communication in chat tools and would not be accepted in face to face situations. Moreover. with interaction users are asked to declare concepts such 
conflicts from the interactions. However, it would be interesting to investigate both approaches concerning a comparison of system derived and user declared conflicts.

Apart from certain concepts that are specific for the turtle puzzle such as the maximum size of the solution, the analysis concepts are supposed to be applicable to diverse problems that can be solved by spatial arrangements of cards. Moreover, other structuring principles of the shared external representation such as the possibility to connect cards by typed links can be easily integrated in the derivation hierarchy in addition to the adjacency relation. The CardBoard user interface provides mechanisms to specify typed connectors between cards to form a visual language, particularly for more open-ended tasks. The approach presented here still has to be evaluated with other problems and with bigger samples of collaboration sessions.

Though the assessment of group interactions is of major interest here, immediate feedback and remediation is technically possible within the framework system. Instead of presenting a rather abstract view of recognized conflicts in an additional analysis workspace, the visualization can easily be modified to represent conflicts directly with the problem solution in the shared workspace in order call the participants attention to it. The system can also be extended by rules that react to frequently occurring conflicts or to unbalanced cooperation and suggest helpful remediation. In addition, it could call the tutors attention to successful collaborations concerning the number of conflicts that occurred.

Currently, we are considering further techniques for task analysis in plan recognition and for analysis-based generalizations of procedures in the context of human-computer interactions for the analysis mechanism. Further work will be dedicated to the extension and refinement of the analysis concepts that are derived. It will be investigated if validation of the concepts can be supported by audio or video protocols. This is expected to be eased by the existing time references in the action protocols that are visualized together with the concepts.

\section{Acknowledgements}

We thank the members of the Collide research group at the University of Duisburg for valuable discussions and helpful suggestions.

\section{Bibliography}

Akhras, F. N. \& Self, J. A. (1996). A process-sensitive learning environment architecture. In C. Frasson, G. Gauthier \& A. Lesgold (editors), Proceedings of the Third International Conference on Intelligent Tutoring Systems ITS-96 (pages 430-438). Berlin, Germany: Springer.

Akhras, F. N. \& Self, J. A. (1999). Modelling the process, not the product, of learning. In S. P. Lajoie (editor), Computers as cognitive tools: Theory change, paradigm shifts and their influence on the use of computers for instructional purposes (volume 2). Mahwah, NJ: Lawrence Erlbaum.

Baker, M. J. \& Lund, K. (1996). Flexibly structuring the interaction in a CSCL environment. In P. Brna, A. Paiva \& J. Self (editors), Proceedings of the European Conference on Artificial Intelligence in Education (pages 401-407). Lisbon, Portugal.

Burton, M., Brna, P., \& Pilkington, R. (1997). Splitting the collaborative atom: How to support learning about collaboration. In B. du Boulay \& R. Mizoguchi (editors), Artificial

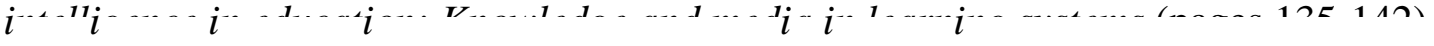


Dillenbourg, P., Baker, M., Blaye, A., \& O'Malley, C. (1995). The evolution of research on collaborative learning. In H. Spada and P. Reimann (eds.), Learning in Humans and Machines, Elsevier Science.

Hoppe, H. U. (1993). Intelligent user support based on task models. In M. SchneiderHufschmidt, T. Kühme, \& U. Malinkowski (editors), Adaptive user interfaces (167--181). North-Holland: Elsevier-Science.

Huhns, M. N. \& Singh, M. P. (editors). (1997). Readings in agents. Palo Alto, CA: Morgan Kaufmann.

Kieras, D. (1994). A guide to GOMS task analysis. University of Michigan.

Lewis, C. (1988). Why and how to learn why: Analysis-based generalization of procedures. Cognitive Science, 12, 211--256.

Mühlenbrock, M., Tewissen, \& Hoppe, H. U. (1997). A framework system for intelligent support in open distributed learning environments. In B. du Boulay \& R. Mizoguchi (editors), Artificial intelligence in education: Knowledge and media in learning systems (pages 191198). Amsterdam, The Netherlands: IOS Press.

Mühlenbrock, M., Tewissen, \& Hoppe, H. U. (1998). A framework system for intelligent support in open distributed learning environments. International Journal of Artificial Intelligence in Education, 9, 256-274.

Roschelle, J. \& Teasley, S. D. (1991). The construction of shared knowledge in collaborative problem solving. In C. O'Malley (editor), Computer supported collaborative learning (pages 67-97). Berlin, Germany: Springer.

Soller, A., Linton, F., Goodman, B. \& Lesgold, A. (1999). Toward intelligent analysis and support of collaborative learning interaction. In Lajoie, S. P. \& Vivet, M. (eds.) Artificial Intelligence in Education - Open Learning Environments: New Computational Technologies to Support Learning, Exploration and Collaboration. (pages 75-82), IOS Press: Amsterdam.

Vosniadou, S. (1996). Towards a revised cognitive psychology for new advances in learning and instruction. Learning and Instruction, 6 (2), 95-109.

\section{Authors' addresses}

Martin Mühlenbrock (muehlenbrock@informatik.uni-duisburg.de)

University of Duisburg, Department of Mathematics/Computer Science, 47048 Duisburg, Germany, Tel (+49) 203379 3554, Fax (+49) 2033793557

Ulrich Hoppe (hoppe@informatik.uni-duisburg.de)

University of Duisburg, Department of Mathematics/Computer Science, 47048 Duisburg, Germany, Tel (+49) 203379 3553, Fax (+49) 2033793557 